CERN-TH/97-338

UPR-0779-T

IEM-FT-166/97

hep-th/9711178

\title{
Classification of Flat Directions in Perturbative Heterotic Superstring Vacua with Anomalous $U(1)$
}

\author{
Gerald Cleaver $^{\dagger}$, Mirjam Cvetič ${ }^{\dagger}$, Jose R. Espinosa* ${ }^{* \dagger}$, Lisa Everett ${ }^{\dagger}$, and Paul Langacker ${ }^{\dagger}$ \\ ${ }^{\dagger}$ Department of Physics and Astronomy \\ University of Pennsylvania, Philadelphia PA 19104-6396, USA \\ ${ }^{*}$ CERN, TH Division \\ CH-1211 Geneva 23, Switzerland
}

\begin{abstract}
We develop techniques to classify $D$ - and $F$-flat directions for $N=1$ supersymmetric string vacua of the perturbative heterotic string theory, which possess an anomalous $U(1)$ gauge group at the tree level. Genus-one corrections generate a Fayet-Iliopoulos term for the $D$-term of $U(1)_{A}$, which is canceled by non-zero vacuum expectation values (VEVs) of certain massless multiplets in such a way that the anomalous $U(1)$ is broken, while maintaining the $D$ - and $F$-flatness of the effective field theory. A systematic analysis of flat directions is given for non-zero VEVs of non-Abelian singlets, and the techniques are illustrated for a specific model. The approach sets the stage to classify the $D$ - and $F$-flat directions for a large class of perturbative string vacua. This classification is a prerequisite to address systematically the phenomenological consequences of these models.
\end{abstract}

CERN-TH/97-338

November 1997 


\section{INTRODUCTION}

As a part of a broader program to address phenomenological implications of string models one is faced with a myriad of challenges, among others the degeneracy of string vacua, origin of supersymmetry breaking, and for some string vacua calculability of the effective Lagrangian.

Nevertheless, one can take a more modest approach to consider a class of specific superstring vacua which at the string scale $\left(M_{\text {string }}\right)$ possess $N=1$ supersymmetry, the standard model (SM) gauge group $S U(2)_{L} \times U(1)_{Y} \times S U(3)_{C}$, as a part of the full gauge structure, and a particle content that includes three ordinary families and at least two SM Higgs doublets; i.e., such superstring vacua possess at least the ingredients of the minimal supersymmetric standard model (MSSM). These models may provide candidate vacua with specific phenomenological predictions, consistent with the MSSM.

Within the perturbative heterotic superstring, classes of such quasi-realistic superstring models were constructed from orbifold constructions [1] with Wilson lines [2, 3], Calabi-Yau compactifications [4], free-fermionic constructions [5 7], covariant lattices [8] and GepnerKazama-Suzuki models [9]. A set of these models constitutes a starting point to address their detailed phenomenological analysis. In general they possess the following features:

- Gauge Group Structure. Along with the SM gauge group there is a non-Abelian hidden sector group and a number of additional $U(1)$ 's, one of them generically anomalous. The hidden sector non-Abelian gauge group may play a role in dynamical supersymmetry breaking.

The $U_{Y}$ is determined as a linear combination of the $U(1)$ factors (or possibly broken hidden sector non-Abelian factors), subject to the constraint that the massless spectrum contains the MSSM particles (three ordinary families and two Higgs doublets), and the pairing of the exotic $S U(3)_{C}$ triplets, $S U(2)_{L}$ doublets and exotic singlets of the observable sector . These constraints significantly restrict the allowed values of $U(1)_{Y}$, often imposing a unique choice of $U(1)_{Y}$.

- Particle Spectrum. The mass spectrum is calculable. In addition to the three ordinary families and two SM Higgs doublets, there are generically a large number of exotic massless states. In particular, there are usually a large number of additional massless matter multiplets, which transform non-trivially under the $U(1)$ 's and/or the standard model symmetry, as well as hidden sector states that transform non-trivially under the $U(1)$ 's and the non-Abelian hidden sector gauge group. In some cases these models also possess massless states that are non-Abelian multiplets under both the SM gauge group and the hidden sector non-Abelian gauge group, thus preventing a clear distinction between the observable and the hidden sector of theory.

\footnotetext{
${ }^{1} \mathrm{~A}$ weaker requirement is that the exotic particles are paired with respect to $S U(3)_{C}$ and the electric charge quantum number, so that there will be no exactly massless colored or electrically charged fermions in the theory. For this weaker requirement there can be exotic states that are chiral under $S U(2)_{L}$ and/or $U(1)_{Y}$, but can acquire masses at the electroweak breaking scale.
} 
- Effective Lagrangian. In these models the couplings of the effective Lagrangian are calculable. In particular, the conformal field theory techniques to calculate the Yukawa couplings as well as higher order couplings in the superpotential have been developed. However, in some cases detailed phenomenological analysis requires determination of terms of very high order (sometimes to all orders) in the superpotential, which is often beyond calculable reach $\mathrm{H}$.

To address the phenomenology of this class of models, the strategy is as follows 8 :

- Step I. Due to the anomalous $U(1)$ gauge symmetry at genus-one there is an additional contribution $\propto M_{\text {string }}^{2} /\left(192 \pi^{2}\right) \operatorname{Tr} Q_{A}$ 14,15 to the corresponding $D$-term (Fayet-Iliopoulos term). The contribution of such a term is canceled [14 16] by giving non-zero vacuum expectation values $(\mathrm{VEVs})$ of $\mathcal{O}\left(M_{\text {string }}\right)$ to certain massless multiplets in such a way that the anomalous $U(1)$ is broken, while maintaining the $D$ - and $F$-flatness of the effective theory. Namely, a "deficiency" in the string construction that produced the anomalous $U(1)$ is remedied at genus-one, by providing a mechanism to "restabilize" the vacuum. A comprehensive analysis of the $D$ - and $F$-flat directions for this class of models is thus a prerequisite to address fully their phenomenological consequences.

- Step II. As the next step, the surviving gauge group, the massless particle quantum numbers and their couplings in the effective Lagrangian at $M_{\text {string }}$ for the restabilized string vacua should be analyzed.

- Step III. Finally, the implications of these models for the low energy phenomenology should be addressed. In this case one faces the problem that the origin of the supersymmetry breaking in string theory is not well understood, and thus one is lacking predictive power for the supersymmetry breaking parameters in the effective Lagrangian. Nevertheless, one may parameterize this ignorance by introducing soft supersymmetry breaking mass terms, and analyze the low energy consequences as a function of these parameters.

The purpose of this paper is to describe a systematic approach which enables one to classify the $D$ - and $F$-flat directions which restabilize the superstring vacuum (Step I). In

\footnotetext{
${ }^{2}$ In general, one cannot classify the non-zero couplings just by using the gauge symmetries of the effective field theory, because certain gauge-allowed terms are absent due to the string dynamics. In certain instances such constraints can be obtained by applying selection rules for the corresponding string amplitudes, as developed for orbifold [10] and blown-up orbifold compactifications [11]. See also [3].

${ }^{3}$ Certain low energy phenomenological consequences can be obtained by addressing only parts of the analysis described below. In particular, taking this limited route, consequences for additional neutral gauge bosons in this class of models were extensively addressed in 12,13.
} 
particular, the analysis focuses on $D$ - and $F$-flat directions with non-zero VEVs for the nonAbelian singlets, only f. (For the sake of completeness an analysis of the same issue including non-Abelian fields as well is needed. However, we postpone this problem for further study.) While the method discussed in this paper is general, and applies to a general perturbative heterotic string model with an anomalous $U(1)$, we chose to illustrate the results for a particular model, namely Model 5 of [7]. This serves as an instructive example, in which the classification of flat directions is particularly simple.

In a subsequent paper [17], the method is applied to the analysis of the flatness constraints for a larger class of models. In later papers we plan to address the phenomenological consequences of these models in detail (Steps II and III).

This paper is organized as follows. In Section II some of the technical features, in particular those associated with the anomalous $U(1)$ for the superstring models (based on the fermionic construction), are discussed. In Sections III and IV the approaches to determine $D$-flat directions for the non-anomalous $U(1)$ 's and anomalous $U(1)_{A}$ are discussed, respectively. In Section V F-flatness constraints are analyzed. The summary and conclusions are given in Section VI.

\section{STRINGY $S U(3)_{C} \times S U(2)_{L} \times U(1)_{Y}$ MODELS}

Gauge structures for quasi-realistic four-dimensional heterotic string models follow the general form,

$$
\left\{S U(3)_{C} \times S U(2)_{L} \in \mathcal{G}\right\}_{\mathrm{obs}} \times \tilde{\mathcal{G}}_{\mathrm{hid}}^{\mathrm{NA}} \times \prod_{n} U(1)_{n} \times \prod_{p} \tilde{\Delta}_{p},
$$

where $\mathcal{G}$ denotes a possible SM GUT or semi-GUT embedding which may include some particular $U(1)$ factors, and $\tilde{\mathcal{G}}_{\text {hid }}^{\mathrm{NA}}$ contains the non-Abelian hidden sector gauge factors. Both observable and hidden $U(1)$ are included in $\prod_{n} U(1)_{n}$, while $\prod_{p} \tilde{\Delta}_{p}$ denotes remaining local discrete symmetries.

"Cracks in the wall" separating the observable and hidden sectors can appear in two forms. The first is when massless states appear as non-trivial representations of both the observable and hidden sector non-Abelian gauge groups (i.e, as "mixed" states). Model 5 of (77) gives an example of such a mixed state that carries $S U(2)_{L} \times S U(2)_{\text {hid }}$ charge. In this case part (or possibly all) of the so-called "hidden sector" is not actually hidden, unless the mixed states acquire string scale masses. If string scale masses are not acquired, a gauge-mediated process may be the dominant form of observable sector supersymmetry breaking.

Second, there may be "shadow" sector $U(1)$ 's, whose charges are carried by both observable and hidden sector states. Although states with both shadow and hidden sector charge are generically present in free fermionic models, acceptable phenomenology requires that such states have masses $m \geq m_{\text {susy }}$. Furthermore, traditional supergravity-mediated supersymmetry breaking suggests that such states should have masses $m \approx M_{\text {string. }}$. Both types of

\footnotetext{
${ }^{4} \mathrm{~A}$ number of specific examples of such $D$ - and $F$-flat directions exist in the literature [3, 6]. However, a comprehensive approach to this problem is lacking.
} 
communication between observable and hidden sectors can have profound phenomenological implications.

The SM hypercharge, $Y$, may be totally embedded in a (semi-)GUT $\mathcal{G}$ or may also have contributions from various $U(1)_{n}$. Typically, some of the extra $U(1)_{n}$ in four-dimensional string models are anomalous. By this we mean those Abelian symmetries have mixed $U(1)$ gravitational anomalies and thus have a non-zero charge trace. For example, to date all known free fermionic four-dimensional $S U(3)_{C} \times S U(2)_{L} \times U(1)_{Y}$ models with extra, unembedded, $U(1)$ factors contain this type of anomaly.

In the initial charge basis, generally more than one $U(1)_{n}$ will appear anomalous. However, the total anomaly from all $U(1)_{n}$ can be rotated into a single $U(1)_{\mathrm{A}}$, defined by

$$
U(1)_{\mathrm{A}} \equiv c_{A} \sum_{n}\left\{\operatorname{Tr} Q_{n}\right\} U(1)_{n}
$$

with $c_{A}$ a normalization coefficient. Following rotation (2), an orthogonal basis $\left\{U(1)_{a}\right\}$ may be chosen for the non-anomalous components of the original set of $U(1)_{n}$.

As a result of the charge trace relationships, generally known as the universal GreenSchwarz (GS) relation,

$$
\frac{1}{k_{m} k_{A}^{1 / 2}} \operatorname{Tr}_{G_{m}} T(R) Q_{A}=\frac{1}{3 k_{A}^{3 / 2}} \operatorname{Tr} Q_{A}^{3}=\frac{1}{k_{a} k_{A}^{1 / 2}} \operatorname{Tr} Q_{a}^{2} Q_{A}=\frac{1}{24 k_{A}^{1 / 2}} \operatorname{Tr} Q_{A} \equiv 8 \pi^{2} \delta_{\mathrm{GS}},
$$

invoked by stringy modular invariance constraints, rotation (2) removes all $U(1)$ triangle anomalies except those involving $U(1)_{A}$ [14. Here $k_{m}$ is the level of the gauge group $G_{m}$ and $2 T(R)$ is the index of the representation $R$. The physical content of eq. (3) is that the related mixed anomalies are canceled by the pseudoscalar partner of the dilaton, which couples universally to all gauge groups. A similar set of anomaly-free $U(1)_{a}$ trace relations indicates that triangle anomalies involving $Q_{A}^{2}$ cannot occur,

$$
\frac{1}{k_{m} k_{a}^{1 / 2}} \operatorname{Tr}_{G_{m}} T(R) Q_{a}=\frac{1}{3 k_{a}^{3 / 2}} \operatorname{Tr} Q_{a}^{3}=\frac{1}{k_{A} k_{a}^{1 / 2}} \operatorname{Tr} Q_{A}^{2} Q_{a}=\frac{1}{24 k_{a}^{1 / 2}} \operatorname{Tr} Q_{a}=0 .
$$

Relations analogous to (3) and (स) involving $Q_{a} Q_{b}, Q_{a} Q_{b} Q_{c}$, etc., also hold.

After rotation of all anomalies into a single $U(1)_{A}$ via (2), the standard anomaly cancelation mechanism [14 breaks $U(1)_{\mathrm{A}}$. However, this occurs at the expense of generating a Fayet-Iliopoulos (FI) D-term,

$$
\mathrm{e}^{\phi} M_{P l}^{2} \delta_{\mathrm{GS}}=\frac{\mathrm{e}^{\phi} M_{P l}^{2}}{192 \pi^{2} k_{A}^{1 / 2}} \operatorname{Tr} Q_{A}
$$

\footnotetext{
${ }^{5}$ Recently, though, an anomaly-free semi-GUT with $S U(4)_{C} \times S U(2)_{L} \times S U(2) \times U(1)^{4} \times\left[E_{7} \times\right.$ $S U(2)]_{\text {hid }}$ gauge group containing observable sector chiral reps was found 18. For a discussion on anomalous $U(1)$ 's in orbifold compactifications see [19].

${ }^{6}$ This rotation is not necessarily sufficient to remove all triangle anomalies from the $U(1)$ orthogonal to $U(1)_{A}$ for general field theoretic models or strongly coupled strings, since the GS relations (3) need not hold then.
} 
where $\phi$ is the dilaton. $g \equiv \mathrm{e}^{\phi / 2}$ is the physical four-dimensional gauge coupling. The FI $D$ term is a genus-one string effect (one-loop effect in the effective field theory) and is therefore calculable in perturbative string theory. It must be canceled by appropriate VEVs of the scalar components $\varphi_{i}$ of supermultiplets $\Phi_{i}$ carrying the anomalous charge so that',

$$
D_{\mathrm{A}}=\sum_{i} Q_{i}^{(A)}\left|\varphi_{i}\right|^{2}+\frac{g^{2} M_{P l}^{2}}{192 \pi^{2}} \operatorname{Tr} Q_{\mathrm{A}}=0 .
$$

$D$-flatness in the non-anomalous directions, along with $F$-flatness, impose additional constraints on the allowed directions of these VEVs,

$$
\begin{aligned}
D_{a} & =\sum_{i} Q_{i}^{(a)}\left|\varphi_{i}\right|^{2}=0, \\
F_{i} & =\frac{\partial W}{\partial \Phi_{i}}=0 ; W=0 .
\end{aligned}
$$

The presence of an anomalous $U(1)_{A}$ can have profound effects on a model including: (i) Generation of a Fayet-Iliopoulos $D$-term and gauge rank reduction via induced VEVs canceling the FI term [3]. (ii) The induced VEVs can eliminate many particles from the low energy spectrum. (iii) It can play a role in explaining fermion mass hierarchies [20] and addressing the $\mu$ problem [21]. Other possible implications are discussed in [22 25].

The model we use to illustrate our general analysis is model 5 of ref. [0], to which we refer for more details. The gauge group is

$$
\left\{S U(3)_{C} \times S U(2)_{L}\right\}_{\mathrm{obs}} \times\left\{S U(4)_{2} \times S U(2)_{2}\right\}_{\mathrm{hid}} \times U(1)_{A} \times U(1)^{6},
$$

and the particle content includes, besides the MSSM multiplets, additional chiral superfields:

$$
\begin{aligned}
& 6(1,2,1,1)+(3,1,1,1)+(\overline{3}, 1,1,1)+ \\
& 4(1,2,1,2)+ \\
& 2(1,1,4,1)+10(1,1, \overline{4}, 1)+8(1,1,1,2)+ \\
& 5(1,1,4,2)+(1,1, \overline{4}, 2)+8(1,1,6,1)+3(1,1,1,3)+ \\
& 42(1,1,1,1),
\end{aligned}
$$

where $(a, b, c, d)$ indicates the representation under $\left(S U(3)_{C}, S U(2)_{L}, S U(4)_{2}, S U(2)_{2}\right)$. In Table I we list the 45 (including three generations of $e^{+}$and $\nu^{c}$ ) non-Abelian singlets of the model with their $U(1)$ charges. A prime on a superfield $S_{i}$ indicates the existence of another superfield $S_{i}^{\prime}$ with the same charges. Some fields $S_{j}$ have a mirror copy $\bar{S}_{j}$ with opposite $U(1)$ charges, and are indicated with a $\sqrt{ }$.

Phenomenological considerations lead to the hypercharge definition [7]

$$
Y=\frac{1}{96}\left(-8 Q_{2}-3 Q_{3}-8 Q_{4}-Q_{5}+Q_{6}\right)
$$

[normalized to give $Y$ (quark doublet $)=1 / 6]$. In following sections we will examine those flat directions involving only non-Abelian singlets with $Y=0$.

\footnotetext{
${ }^{7}$ Our convention for defining $D_{\mathrm{A}}$ is that the corresponding $D$ term in the Lagrangian is $\frac{1}{2 k_{A}} g^{2} D_{\mathrm{A}}^{2}$, and similarly for $D_{a}$.
} 


\section{D-FLAT DIRECTIONS FOR THE NON-ANOMALOUS $U(1)$ 'S}

Any flat direction must have vanishing $D$-flat terms for all the non-anomalous $U(1)$ factors of the gauge group. We consider first the space of such flat directions, leaving aside for the moment the questions of vanishing anomalous $D$-term and $F$-flatness. In the rest of this section then, flat direction will refer to a direction with zero $D$-terms for the nonanomalous $U(1)$ 's.

A powerful and convenient way of analyzing the moduli space of flat directions in a given model makes use of the correspondence between the holomorphic gauge-invariant monomials built out of the chiral fields and the $D$-flat directions [26 29]. In the absence of a superpotential [and of an anomalous $U(1)$, see next section], the set of all such monomials describes the variety of classical supersymmetric vacua of the model. When the superpotential does not vanish, the moduli space is described by imposing $F$-flatness constraints on the holomorphic monomials.

We restrict our discussion to those flat directions exciting only chiral superfields $\Phi_{i}$ (with scalar components $\varphi_{i}$ ) not charged under any of the non-Abelian gauge groups of a given model. Those fields generically carry non-zero $U(1)_{a}$ charges, $\left[Q_{i}^{(a)}\right.$ for the field $\left.\Phi_{i}\right]$, where we reserve the index $a=A$ for the anomalous $U(1)$ and the rest, $(a=1, \ldots, m)$ to the nonanomalous $U(1)$ 's (that is, we work in the rotated basis). The space of all field directions with zero $D$-terms for $a=1, \ldots, m$ is described by the set of holomorphic $U(1)_{a}$ gaugeinvariant monomials in the $\Phi_{i}$ 's. Such monomials are not necessarily invariant under the anomalous $U(1)$ symmetry, so that they will generically carry a non-zero $U(1)_{A}$ charge.

It may be useful at this point to spell out in more detail how this correspondence works in this simple $U(1)$ case and show explicitly how the flat directions are parameterized in terms of field VEVs. Let $N$ be the number of chiral non-Abelian singlets and let us normalize all $U(1)$ charges so that they are integers. The $m D$-flatness constraints are

$$
D_{a}=\sum_{i} Q_{i}^{(a)}\left|\varphi_{i}\right|^{2}=0, \quad(a=1, \ldots, m) .
$$

Consider now a generic holomorphic invariant monomial (HIM) of the form

$$
P \equiv \Pi_{i}^{\prime} \Phi_{i}=\Pi_{i} \Phi_{i}^{n_{i}}
$$

where the prime indicates that the index $i$ can take the same value several times $\left(n_{i} \geq 0\right)$. The requirement of $U(1)_{a}$ gauge invariance of $P$ reads

$$
\sum_{i}^{\prime} Q_{i}^{(a)}=\sum_{i} n_{i} Q_{i}^{(a)}=0
$$

It is immediately obvious that the choice of VEVs

$$
\left|\varphi_{i}\right|^{2}=n_{i}|\psi|^{2}
$$

satisfies $D_{a}=0$ automatically for arbitrary $|\psi|$. If the monomial $P$ is actually built as the product of two other HIM's 


$$
P=N_{1} N_{2}=\left[\Pi_{i} \Phi_{i}^{n_{i}^{(1)}}\right]\left[\Pi_{j} \Phi_{j}^{n_{j}^{(2)}}\right],
$$

the VEVs can be chosen as

$$
\left|\varphi_{i}\right|^{2}=n_{i}^{(1)}\left|\psi_{1}\right|^{2}+n_{i}^{(2)}\left|\psi_{2}\right|^{2}
$$

with both $\left|\psi_{1}\right|$ and $\left|\psi_{2}\right|$ as arbitrary parameters (giving a multidimensional flat direction). It is clear then how HIM's correspond to $D$-flat directions. The converse is also true. Given a solution to the $m$ eqs. (12), suppose no subset of the fields with non-zero VEVs satisfy the same system of equations (if there are, then we analyze each subset in turn). This assumption implies that the field VEVs are related to each other by a relation of the form $\left|\varphi_{j}\right|^{2}=r_{j}|\psi|^{2}$ with $r_{j} \geq 0$ and

$$
\sum_{j} Q_{j}^{(a)} r_{j}=0
$$

Each $Q_{j}^{(a)}$ is an integer number by our choice of normalization. Taking $r_{1}$ as an integer and having assumed that the solution to the $D$-flat constraints cannot be split in sub-solutions, a theorem of elementary algebra [30] tells that the $r_{j}$ 's must be rational. Rescaling them as integers, we can then construct the holomorphic invariant monomial

$$
P=\prod_{j} \Phi_{j}^{r_{j}}
$$

associated with the particular solution of the $D$-flat constraints.

If the original solution $P$ could be decomposed in subsets of solutions, then the corresponding monomial would be a product of simpler invariant monomials, associated as above with each separate subsolution. Flat directions which cannot be decomposed in such a way would be one-dimensional (they will depend on a single arbitrary VEV $\psi$ ). As a general rule, the (complex) dimension of a flat direction $P$ will be equal to the number of different fields excited minus the number of $D$ constraints, as given by $\left.\operatorname{Rank}\left[Q_{i}^{(a)}\right]\right|_{P}$, where $Q_{i}^{(a)}$ denotes the full $N \times m$ charge matrix and $\left.\right|_{P}$ means that only the rows corresponding to fields present in $P$ are selected to compute the rank. This rank is the number of $U(1)$ 's which are broken along the flat direction $P$. In other words, the initial number of degrees of freedom, as given by the number of different fields in $P$, equals the dimension of the flat direction plus the number of Goldstone bosons:

$$
\text { [\# different fields in } P]=\operatorname{dim} P+\left[\# \text { broken } U(1)^{\prime} s\right] \text {. }
$$

We next discuss the dimension of the space of $D$-flat directions. The starting field space is an $N$-dimensional complex space. Any one-dimensional HIM (or equivalently, any onedimensional solution of the $D$-flat constraints)

$$
P=\Pi_{i} \Phi_{i}^{r_{i}}, \quad\left(r_{i} \geq 0\right)
$$

can be associated with a vector in this $N$-dimensional field space proportional to

$$
v_{P}=\left(r_{1}, \ldots, r_{i}, \ldots, r_{N}\right),
$$


with a constant of proportionality equal to the free $\mathrm{VEV}|\psi|^{2}$. It is then clear that any flat direction can be expressed as a linear combination of such elementary vectors 8 and one can construct a basis of that space by finding a collection $\left\{v_{\alpha}\right\}$ of linearly independent onedimensional flat directions. That basis, which has a finite number $d$ of elements $(d \leq N)$, will be most useful for the description of the space of flat directions.

In general, the dimension of that space is equal to the dimension $N$ of the original field space minus the number of independent constraints imposed by the $m D_{a}=0$ conditions (these are real conditions but $m$ phases are fixed by gauge fixing). This would give in principle $d=N-m$ for the dimension of the moduli space of flat directions. If not all $D_{a}=0$ conditions on the $\Phi_{i}$ are independent [the number of independent conditions is the rank of the $N \times m$ charge matrix $\left.Q_{i}^{(a)}\right]$ the dimension of the moduli space can be larger. In general,

$$
d \equiv \operatorname{dim} \mathcal{M} \leq N-\operatorname{Rank}\left[Q_{i}^{(a)}\right] .
$$

In some cases the inequality is not saturated. This can only happen if under some $U(1)$ (or linear combination of them) all the non-Abelian singlet fields have $Q_{i} \geq 0$ or $Q_{i} \leq 0$. There is no way of constructing an HIM that includes those fields with non-zero charges under that particular $U(1)$ so that they will never enter in any flat-direction?. Such fields may as well be removed from the list of $N$ fields for the purpose of discussing the space of flat directions. After such fields have been removed, one ends up with $N^{*} \leq N$ fields and

$$
d \equiv \operatorname{dim} \mathcal{M}=N^{*}-\operatorname{Rank}\left[Q_{i}^{(a)}\right]^{*},
$$

and $\operatorname{Rank}\left[Q_{i}^{(a)}\right]^{*}\left(\leq \operatorname{Rank}\left[Q_{i}^{(a)}\right]\right)$ is the rank of the $N^{*} \times m^{*}$ charge matrix of the $N^{*}$ remaining fields [which have non-zero charges under $m^{*} \leq m U(1)$ 's].

Once this basis $\left(\left\{M_{\alpha}\right\}\right.$ HIM's with $\left.\alpha=1, \ldots, d\right)$ has been constructed, any flat direction $P$ can be expressed in terms of the $M_{\alpha}$ 's as

$$
P^{n}=\Pi_{\alpha} M_{\alpha}^{n_{\alpha}}
$$

\footnotetext{
${ }^{8}$ Not every linear combination of these elementary vectors will correspond to a flat direction though, because of the constraint $r_{i} \geq 0$, which renders the problem non-trivial. In a linear combination of these vectors, $v=\sum_{\kappa} a_{\kappa} v_{P_{\kappa}}$, negative coefficients $a_{\kappa}$ are allowed provided the vector $v$ also has $r_{i} \equiv \sum_{\kappa} a_{\kappa} r_{i \kappa} \geq 0$ (see example at the end of this section).

${ }^{9} \mathrm{As} \operatorname{Tr} U(1)^{(a)}=0$ over all the fields in the model, this class of fields exists only when we restrict our attention to a particular sector of the model. Such fields can enter in flat directions that excite fields outside that sector.

${ }^{10} P^{n}$ by itself is exactly the same flat direction as $P$; the power is only important when relating $P$ to other HIM's.
} 
where $n$ and $n_{\alpha}$ are integers, with $n \geq 1$ but $n_{\alpha}$ can take both signs $\amalg$. Such a basis contains in a compact form all the information required about the classical moduli space.

We will present now such a basis for our model. In principle, this basis is not uniquely determined and different choices can be made which are more or less convenient for different purposes. One simple procedure to generate the basis is to make an automated search of HIM's, retaining only those which are independent, until the number of them saturates the dimension of the moduli space. An alternative and more systematic way of generating the basis is to form first a basis of invariants for the first $U(1)$ group. These invariants are then combined to form an invariant basis for the second $U(1)$ factor and so on. This procedure is simple because the construction of invariants of a single $U(1)$ is trivial. However, the elements of the final basis generated in this way are usually more complicated. In table II, we report two different bases for our example model (for flat directions built out of $Y=0$ singlet fields only). An element like $\left\langle 8,1^{2}\right\rangle$ stands for the HIM

$$
M_{6}=\Phi_{8} \Phi_{1}^{2}
$$

Basis A is constructed in such a way as to minimize the number (and power) of the fields entering the basis elements. Basis B will be suitable for some future discussions. Of course, all the results concerning flat directions are independent of which particular basis is used.

In our model, after selecting $Y=0$ singlet fields only, we have 20 fields plus 10 copies, and $\operatorname{Rank}\left[Q_{i}^{(a)}\right]=6-1$ (6 initial non-anomalous $U(1)$ 's minus hypercharge, as the hypercharge $D$-term vanishes trivially for $Y=0$ fields). The dimension of the $D$-flat space is then $d=25$. In the table, we show only those elements involving the 20 different fields, so that we have only 15 elements. The 10 remaining elements not shown are obtained trivially by appropriate substitution of some fields by their copies.

To clarify the meaning of eq. (25), we give an example of a flat direction which is not contained in the bases of Table II and requires some negative $n_{\alpha}$ :

$$
P \equiv\langle 22,8, \overline{5}\rangle=\frac{M_{13} M_{9} M_{4}}{M_{11} M_{2}}=\frac{\langle 15,8, \overline{3}\rangle\langle 22,18,3\rangle\langle 5, \overline{5}\rangle}{\langle 18,15,5\rangle\langle 3, \overline{3}\rangle},
$$

or, in terms of the vectors of the form (22):

$$
v_{P}=v_{13}+v_{9}+v_{4}-v_{11}-v_{2} .
$$

Although knowledge of the basis of $D$-flat directions is very convenient, it proves useful to have, in addition, a list of all one-dimensional flat directions. The number of them can be much larger than the dimension $d$ of the moduli space but is finite. In particular, from (20), it is clear that there is an upper limit on the number of different fields that can appear in a onedimensional flat direction. Using such a superbasis, every flat direction can be factorized in terms of one-dimensional elements in an expression similar to (25) but with $n_{\alpha} \geq 0$. This simplifies considerably the analysis of the moduli space as only trivial multiplications

\footnotetext{
${ }^{11} \mathrm{An}$ equivalent description of eq. (25) is that the vector $v_{P}$ associated with the direction $P$ can always be expressed as a linear combination of the basis vectors $v_{\alpha}$.
} 
of one-dimensional HIM's are involved and positivity constraints on the VEV-squares are automatically satisfied. For example, one is usually interested in finding if particular flat directions exist with non-zero VEVs for some given fields while keeping zero VEVs for some other fields. In principle, the use of the $d$-dimensional basis is sufficient to answer such questions, but it can become cumbersome in practice (especially for large dimensional bases, as is usually the case). In contrast, by simple inspection of the superbasis one is able to determine whether such directions do exist. The usefulness of such a list will become clear in the next sections, especially for the discussion of $F$-flatness. In Table III we present such a superbasis for our model example. Only the 73 directions involving non-primed fields are shown as the rest are obtained trivially from these.

\section{ANOMALOUS $U(1)$}

In the presence of an anomalous $U(1)$ one should further require the vanishing of the corresponding $D$-term, which now includes a Fayet-Iliopoulos term:

$$
D_{A}(P)=\sum_{i} Q_{i}^{A}\left|\varphi_{i}\right|^{2}+\xi
$$

with

$$
\xi=\frac{g^{2} M_{P l}^{2}}{192 \pi^{2}} \operatorname{Tr} Q_{A} \neq 0
$$

For the analysis of the flat directions in the presence of an anomalous $U(1)$ it is useful to find subsets of chiral fields for which the anomalous $U(1)$ is a linear combination of the non-anomalous $U(1)$ 's, say

$$
Q_{j}^{A}=\bar{Q}_{j} \equiv \sum_{a=1}^{m} \alpha_{a} Q_{j}^{(a)},
$$

where the $\alpha_{a}$ can be chosen for convenience. Obviously, such a relation cannot hold for all the chiral fields in the model. We will choose the $\alpha_{a}$ trying to maximize the number of fields

for which $\bar{Q}$ equals the anomalous charge $Q_{A}$. It is remarkable that in all the models we have studied, a definition exists which matches the anomalous charges of a very large number of fields. This observation helps considerably in the analysis and search of flat directions.

Defining the quantities

$$
\hat{Q}_{j}=Q_{j}^{A}-\bar{Q}_{j}
$$

we can classify all the chiral fields in three different types, depending on the sign of $\hat{Q}_{j}$ :

$$
\begin{aligned}
& \Phi_{j}^{+}, \text {if } \hat{Q}_{j}>0 \\
& \Phi_{j}^{0}, \quad \text { if } \hat{Q}_{j}=0 \\
& \Phi_{j}^{-}, \quad \text { if } \hat{Q}_{j}<0 .
\end{aligned}
$$


We can extend the definition of $\hat{Q}$ from the fields to the flat directions. If the flat direction $P$ is defined by (25), then

$$
\hat{Q}\left(P^{n}\right)=n \hat{Q}(P)=\sum_{\alpha} n_{\alpha} \hat{Q}\left(M_{\alpha}\right)
$$

and

$$
\hat{Q}\left(M_{\alpha}=\Pi_{i} \Phi_{i}^{n_{i}}\right)=\sum_{i} n_{i} \hat{Q}_{i}
$$

By invariance of $P$ under the non-anomalous $U(1)$ 's, $\hat{Q}(P)$ turns out to be precisely the anomalous charge of $P$.

The sign of the Fayet-Iliopoulos term $\xi$ determines which fields can actually form a flat direction. The statement is:

If $\xi>0(<0)$, any flat direction must contain at least one of the fields $\Phi_{j}^{-}\left(\Phi_{j}^{+}\right)$.

The proof goes as follows: First, if the flat direction is of the form

$$
P^{0}=\Pi_{i}^{\prime} \Phi_{i}^{0}
$$

one has

$$
D_{A}\left(P^{0}\right)=\sum_{i} Q_{i}^{A}\left|\varphi_{i}^{0}\right|^{2}+\xi=\sum_{i} \sum_{a=1}^{m} \alpha_{a} Q_{i}^{(a)}\left|\varphi_{i}^{0}\right|^{2}+\xi=\sum_{a=1}^{m} \alpha_{a} D_{a}\left(P^{0}\right)+\xi=\xi \neq 0 .
$$

For a flat direction of the form

$$
P^{ \pm}=\left(\Pi_{i}^{\prime} \Phi_{i}^{0}\right)\left(\Pi_{j}^{\prime} \Phi_{j}^{ \pm}\right)
$$

one has instead

$$
D_{A}\left(P^{ \pm}\right)=\sum_{i} \sum_{a=1}^{m} \alpha_{a} Q_{i}^{(a)}\left|\varphi_{i}^{0}\right|^{2}+\xi+\sum_{j} Q_{j}^{A}\left|\varphi_{j}^{ \pm}\right|^{2}=\xi+\sum_{j} \hat{Q}_{j}\left|\varphi_{j}^{ \pm}\right|^{2} .
$$

If $\xi>0$, the only possible way of canceling the $\xi$ term is by having negative $\hat{Q}_{j}$ 's. A similar consideration applies for $\xi<0$, which requires some positive $\hat{Q}_{j}$. To cancel the Fayet-Iliopoulos term, some free VEV in the flat direction $P$ (corresponding to some field with the right sign of $\hat{Q}$ ) is fixed in terms of $\xi$ and the dimensionality of $P$ drops by one. The final dimension of $P$ would satisfy eq. (20) if the anomalous $U(1)$ is counted among the broken $U(1)$ 's.

If in some model all the fields which are singlets under the non-Abelian group factors have the wrong value of the $\hat{Q}_{j}$ charges to form a flat direction out of them, the cancelation of the Fayet-Iliopoulos term is necessarily accompanied by the spontaneous breaking of some of the non-Abelian gauge group factors: some non-zero VEV for fields charged under the non-Abelian groups are required.

In table I we also show the values of $\hat{Q}_{j}$ for our example model, with $\bar{Q}$ defined as

$$
\bar{Q}_{j}=-\frac{1}{3} Q_{j}^{(6)}
$$


We see that the only fields with non-zero $\hat{Q}$ are $S_{1}(\hat{Q}=32), \bar{S}_{1}(\hat{Q}=-32)$ and $S_{19}^{\left({ }^{\prime}\right)}$ $(\hat{Q}=-32)$. Then, any flat direction involving only $Y=0$ singlets must necessarily have a non-zero $S_{1} \mathrm{VEV}$ [in this model the trace of the anomalous $U(1)$ is negative].

In Table II we list also the $\hat{Q}$ values for the basis elements. If all the $\hat{Q}$ 's were zero, $Q^{A}$ would be a linear combination of the other $U(1)$ 's in the sector considered. If all $\hat{Q}$ 's for the elements of the basis are either zero or have the wrong sign to cancel $\xi$, one cannot conclude that no flat direction exists with the appropriate sign of $\hat{Q}$ : starting with a $\hat{Q}=0$ direction that contains two fields with opposite $\hat{Q}$, the bad-sign field could be divided out using the wrong sign $\hat{Q}$ basis element. Either by manipulating the elements of the basis or by direct inspection of the full list of one-dimensional flat directions it is easy to find all possible one-dimensional (before canceling $\xi$ ) directions with the correct $\hat{Q}$ to cancel the Fayet-Iliopoulos term. In our example model, from Table III one finds a total of five such directions:

$$
\begin{aligned}
& P_{1} \equiv R_{6}=\left\langle 8,1^{2}\right\rangle, \\
& P_{2} \equiv R_{22}=\left\langle 14, \overline{6}, 3,1^{2}\right\rangle, \\
& P_{3} \equiv R_{23}=\left\langle 18,5,3,1^{2}\right\rangle, \\
& P_{4} \equiv R_{42}=\left\langle 14,5, \overline{4}, 3,1^{2}\right\rangle, \\
& P_{5} \equiv R_{43}=\left\langle 18, \overline{6}, 4,3,1^{2}\right\rangle .
\end{aligned}
$$

To these, one should add those similar monomials obtained by replacing some field by its copy $\left(\bar{S}_{6} \rightarrow \bar{S}_{6}^{\prime}, S_{14} \rightarrow S_{14}^{\prime}, S_{18} \rightarrow S_{18}^{\prime}, S_{5} \rightarrow S_{5}^{\prime}, S_{8} \rightarrow S_{8}^{\prime}\right)$. In general models, the number of different $P_{\alpha}$ 's can be large and even exceed the dimension of the moduli space. To describe the set of all $P_{\alpha}$ 's it is enough to find a subset of independent ones. Combinations of the $P_{\alpha}$ 's in that subset will generate all the $P_{\alpha}$ 's. A convenient basis for all the $D$-flat directions can be arranged that contains this basis for the $P_{\alpha}$ 's as a sub-basis and is completed by other, independent, elements with zero or the wrong sign of $\hat{Q}$. Such a basis for our model is presented as basis B in Table II.

The defining properties of the $P_{\alpha}$ directions are as follows: first, they are one-dimensional (and thus cannot be factorized in simpler HIM's), and second, they have $\hat{Q}$ of the correct sign. These two properties determine the following important result:

Every $D$-flat direction can be written in the form

$$
P^{n}=P_{\alpha} N
$$

with $N$ some HIM (not necessarily of $\hat{Q}>0$ ) and $P_{\alpha}$ one of the five special HIM's listed in (41) (or some version of them involving copies of the fields).

\section{F-FLATNESS}

When the superpotential $W$ is non-vanishing, the effective potential will receive $F$-term contributions that can lift some of the $D$-flat directions we have discussed so far. The conditions to maintain zero potential along a given $D$-flat direction are

$$
F_{i} \equiv \partial W / \partial \Phi_{i}=0 ; W=0,
$$


for all $\Phi_{i}$ in the theory.

Consider a $D$-flat direction

$$
P=\Pi_{i}^{\prime} \Phi_{i}
$$

[with $\hat{Q}(P) \neq 0$ of sign opposite to that of $\operatorname{Tr} Q_{A}$ ]. There are two types of superpotential terms that can lift this generic flat direction. First, there can be terms of the form

$$
W_{A} \sim\left(\prod_{i \in P}^{\prime} \Phi_{i}\right)^{n},
$$

where $\prod_{i \in P}^{\prime} \Phi_{i}$ is a gauge invariant holomorphic monomial formed by a subset of the chiral fields that enter in $P$. These terms will generate a non-zero potential along the flat direction $P$ (barring a cancelation of different contributions to $\left.\partial W_{B} / \partial \Phi_{i}\right)$. A flat direction will be called type-A if such terms are allowed by the gauge symmetries. Note that $P$ itself is not gauge invariant under the anomalous $U(1)$, and so terms $W_{A} \sim P^{n}$ are forbidden by gauge symmetry. This is in contrast with the situation in the absence of an anomalous $U(1)$, in which case the HIM's associated with any flat direction are truly gauge invariant and thus all flat directions are type-A.

Other terms that can lift the generic flat direction $P$ are of the form

$$
W_{B} \sim \Psi\left(\Pi_{i \in P}^{\prime} \Phi_{i}\right),
$$

with $\Psi \notin P$. This term would contribute to the potential the dangerous piece $\left|\partial W_{B} / \partial \Psi\right|^{2}$ which would lift $P$ (again barring cancelations). This exhausts all possible superpotential terms giving a non-zero potential along $P$. As we are examining in this paper the flat directions formed out of non-Abelian singlets only, we can restrict our attention to the superpotential for these fields (gauge invariance requires that $\Psi$ in $W_{B}$ is also a non-Abelian singlet). We will say a flat direction is type-B if only terms of the type $W_{B}$ are allowed by the gauge symmetries. The condition $W=0$ could be violated only by $W_{A}$ terms.

Ideally, one would like to find all directions which are $F$-flat to all orders of the nonrenormalizable superpotential. If the $D$-flat direction is type-A, that would require that some non-gauge symmetry (e.g., some string selection rule derived using conformal field theory) conspires to forbid the infinite number of terms of $W_{A}$ type. If, on the other hand, the flat direction is type-B, only a finite number of $W_{B}$ terms can exist, and knowledge of the superpotential up to some finite order in the nonrenormalizable terms is all that is required to prove $F$-flatness to all orders.

We will restrict our analysis to type-B flat directions (in doing so, we may of course leave out some true flat directions, but proving so is a difficult task). A given $D$-flat direction $P$ will be type-B if no gauge invariant [including $U(1)_{A}$ ] holomorphic monomial can be built out of the fields in $P$. Equivalently, for a type-B $D$-flat direction $P$, one cannot write

$$
P^{n}=N N^{\prime} \quad(n \geq 1),
$$

with $N$ a fully invariant $(\hat{Q}=0)$ holomorphic monomial.

The classification of $D$-flat directions we made in the previous section will prove most useful for finding all type-B flat directions, as we will show now in the case of our particular model. Recall that any $D$-flat direction can be written as 


$$
P^{n}=P_{\alpha}^{\left({ }^{\prime \prime}\right.} N
$$

where the $P_{\alpha}$ 's are listed in (41). There are several cases to consider depending on the anomalous charge of $N$.

- $N=1$. This gives just the list of $P_{\alpha} D$-flat directions. These are type-B directions ${ }^{12}$ because they are one-dimensional, and so cannot be factorized as in (47).

- $N=\Pi_{i}^{\prime} \Phi_{i}$ has $\hat{Q}=0$. Then, $N$ is gauge invariant under all $U(1)$ 's, and the superpotential may contain terms of the form

$$
W_{A} \sim N^{n}
$$

Thus, $P$ would be type-A.

- $N=\Pi_{i}^{\prime} \Phi_{i}$ has $\hat{Q}<0$. To have $\hat{Q}(P)>0$ in (48) it is necessary that $|\hat{Q}(N)|<\hat{Q}\left(P_{\alpha}\right)$. As both $\hat{Q}$ 's can be taken to be integers, the superpotential could contain terms of the form

$$
W_{A} \sim\left(P_{\alpha}^{m} N^{p}\right)^{n}
$$

with $m \hat{Q}\left(P_{\alpha}\right)+p \hat{Q}(N)=0$. This proves $P$ is type-A.

- $N=\Pi_{i}^{\prime} \Phi_{i}$ has $\hat{Q}>0$. Then, using the results of the previous section, we can write again

$$
N=P_{\beta} N^{\prime}
$$

and we should next analyze $N^{\prime}$.

By getting back to the starting point in this way we have proved that all type-B $D$-flat directions must be generated by combining the $P_{\alpha}$ 's alone.

We turn then to the analysis of all possible flat directions built out of the $P_{\alpha}$ 's in our model. Some combinations of $P_{\alpha}$ 's will not be type-B, and we will not consider them further. For the type-B combinations it is simple to find all possible $W_{B}$ type terms and the superbasis is very useful for this purpose. For a given type-B direction $P$, any possible $W_{B}$ term is built of the few elements in the superbasis which involve at most one field not contained in $P$. Usually, it is enough to consider one-dimensional $\hat{Q}=0$ invariants, as multidimensional invariants will generally contain at least two fields (or the same field $\Psi$ to a power larger than one) that do not appear in the type-B flat direction, and thus do not spoil $F$-flatness. By checking whether these terms appear in the superpotential (or are forbidden by stringy

\footnotetext{
${ }^{12}$ One way to check this is to compute $\left.\operatorname{Rank}\left[Q_{i}^{A}, Q_{i}^{(a)}\right]\right|_{P_{\alpha}}$. If this rank equals the number of different fields in $P_{\alpha}$ no gauge-invariant monomial can be built out of the fields in $P_{\alpha}$. However, the existence of an invariant is not guaranteed if the rank is smaller.
} 
arguments) we can determine whether the type-B $D$-flat direction is also $F$-flat to all orders. We then need to know the superpotential to some given order. In ref. [7], $W$ was calculated up to $4^{\text {th }}$ order terms. The non-Abelian singlet part reads ${ }^{\text {T5}}$ :

$$
\begin{aligned}
W_{2} & =0 \\
W_{3} & =S_{4}\left(\bar{S}_{6} \bar{S}_{5}^{\prime}+\bar{S}_{6}^{\prime} \bar{S}_{5}\right)+\bar{S}_{4}\left(S_{5} S_{6}^{\prime}+S_{5}^{\prime} S_{6}\right)+S_{18}\left(S_{5} S_{15}^{\prime}+S_{5}^{\prime} S_{15}\right) \\
& +S_{14}\left(S_{15} \bar{S}_{6}^{\prime}+S_{15}^{\prime} \bar{S}_{6}\right)+S_{23}\left(S_{6} S_{8}^{\prime}+S_{6}^{\prime} S_{8}\right)+\bar{S}_{3}\left(S_{15} S_{8}^{\prime}+S_{15}^{\prime} S_{8}\right) \\
& +S_{22}\left(\bar{S}_{5} S_{8}^{\prime}+\bar{S}_{5}^{\prime} S_{8}\right) \\
W_{4} & =S_{1} S_{8}^{\prime} S_{20} S_{19}^{\prime} .
\end{aligned}
$$

The different Yukawa couplings, of order $g$, are not indicated explicitly, nor is the $\mathcal{O}\left(M_{P l}^{-1}\right)$ coefficient of $W_{4}$. With this information we now examine all possible combinations of the $P_{\alpha}$ 's:

- $P_{1}=\left\langle 8,1^{2}\right\rangle$ and primed versions of it. This is type-B, and the only $W_{B}$ term allowed by gauge symmetries is the mass term

$$
W_{B} \sim S_{1} \bar{S}_{1}
$$

Mass terms do not appear in (52), and we conclude that $P_{1}$ is not only $D$-flat but also $F$-flat to all orders in the nonrenormalizable terms in the superpotential (the same applies to $P_{1}^{\prime}=\left\langle 8^{\prime}, 1^{2}\right\rangle$ and products of both).

- $P_{2}=\left\langle 14, \overline{6}, 3,1^{2}\right\rangle$, etc. are also type-B, with lifting terms

$$
\begin{aligned}
& W_{B}^{(2)} \sim S_{1} \bar{S}_{1}+S_{3} \bar{S}_{3}+S_{6} \bar{S}_{6}+\text { primed copies } \\
& W_{B}^{(3)} \sim S_{15} S_{14} \bar{S}_{6}+S_{23} S_{14} S_{3}+\text { p.c. }
\end{aligned}
$$

We see that $S_{15}^{\prime} S_{14} \bar{S}_{6}$ does appear in (53) and lifts $P_{2}$. However, the directions $P_{2}^{\prime(\prime)}=$ $\left\langle 14^{\prime}, \overline{6}^{(\prime)}, 3,1^{2}\right\rangle$ remain flat to all orders (but $P_{2} P_{2}^{\prime}$ and $P_{2}^{\prime \prime \prime}=\left\langle 14, \overline{6}^{\prime}, 3,1^{2}\right\rangle$ are also lifted).

- $P_{3}=\left\langle 18,5,3,1^{2}\right\rangle$ is type-B with (omitting mass terms)

$$
W_{B}^{(3)} \sim S_{18} S_{15} S_{5}+S_{22} S_{18} S_{3}+\text { p.c. }
$$

Comparing with (53), we find that $S_{18} S_{15}^{\prime} S_{5}$ lifts $P_{3}$ but $P_{3}^{\prime(\prime)}=\left\langle 18^{\prime}, 5^{(\prime)}, 3,1^{2}\right\rangle$ are flat to all orders (while $P_{3} P_{3}^{\prime}$ and $P_{3}^{\prime \prime \prime}=\left\langle 18,5^{\prime}, 3,1^{2}\right\rangle$ are also lifted).

- $P_{4}=\left\langle 14, \overline{4}, 5,3,1^{2}\right\rangle$ and all its primed versions are type-B but are lifted by

$$
W \sim \bar{S}_{4}\left(S_{5} S_{6}^{\prime}+S_{5}^{\prime} S_{6}\right)
$$

\footnotetext{
${ }^{13}$ Those terms in Table III which have $\hat{Q}=0$ but do not appear in $W$ are examples of terms forbidden by conformal selection rules.
} 
- $P_{5}=\left\langle 18, \overline{6}, 4,3,1^{2}\right\rangle$ and all its primed versions are type-B but are lifted by

$$
W \sim S_{4}\left(\bar{S}_{6} \bar{S}_{5}^{\prime}+\bar{S}_{6}^{\prime} \bar{S}_{5}\right) .
$$

- $P_{1} P_{2}=\left\langle 14,8, \overline{6}, 3,1^{4}\right\rangle$ is type-B. Besides those terms already present for $P_{1}$ and $P_{2}$ separately, there are no additional contributions to $W_{B}$. Then, only the combinations with $P_{2}^{\prime(\prime)}$ remain flat to all orders.

- $P_{1} P_{3}$ : same as above.

- $P_{2} P_{3}=\left\langle 18,14, \overline{6}, 5,3^{2}, 1^{4}\right\rangle$ is a particularly interesting type-B flat direction. The dangerous $W_{B}$ terms are just those discussed for $P_{2}$ and $P_{3}$. One way to satisfy the $F$-flatness constraint is to choose adequately the primed copies appearing in the flat direction. For example, $P_{2}^{\prime} P_{3}^{\prime}=\left\langle 18^{\prime}, 14^{\prime}, \overline{6}, 5,3^{2}, 1^{4}\right\rangle$ remains flat to all orders (because $P_{2}^{\prime}$ and $P_{3}^{\prime}$ have zero $F$-terms and there are no mixed terms to worry about). Another interesting possibility is that there is a non-trivial cancelation among different $F$-terms leaving some direction flat. This happens for $P_{2} P_{3}=\left\langle 18,14, \overline{6}, 5,3^{2}, 1^{4}\right\rangle$. The term in the potential (with $\Psi=S_{15}^{\prime}$ ) that would lift this flat direction is

$$
V_{F}=\left|\varphi_{14} \varphi_{\overline{6}}+\varphi_{18} \varphi_{5}\right|^{2} .
$$

The $D$-term constraints would give the relations

$$
\begin{array}{llrl}
\left|\varphi_{1}\right|^{2} & =2 x^{2}, & & \left|\varphi_{6}\right|^{2}=|\psi|^{2}, \\
\left|\varphi_{3}\right|^{2} & =x^{2}, & & \left|\varphi_{14}\right|^{2}=|\psi|^{2}, \\
\left|\varphi_{5}\right|^{2} & =x^{2}-|\psi|^{2}, & & \left|\varphi_{18}\right|^{2}=x^{2}-|\psi|^{2},
\end{array}
$$

with

$$
x^{2}=-\frac{\xi}{64},
$$

and $|\psi|^{2}$ arbitrary (except for the constraint $|\psi|^{2}<x^{2}$ ). It is trivial to see that with a convenient choice of the sign of the VEVs in (60) and fixing $|\psi|^{2}=x^{2} / 2$, one obtains $V_{F}=0$ and thus $F$-flatness to all orders. The same mechanism leaves $P_{2}^{\prime \prime \prime} P_{3}^{\prime \prime \prime}$ flat but cannot work for $P_{2} P_{3}^{\prime \prime \prime}$ or $P_{2}^{\prime \prime \prime} P_{3}$.

- $P_{1} P_{2} P_{3}=\left\langle 18,14,8, \overline{6}, 5,3^{2}, 1^{6}\right\rangle$ is type-B. The $W_{B}$ terms are those of $P_{1}, P_{2}$ and $P_{3}$. One possibility to obtain $F$-flatness is to choose conveniently the primed copies and, for example, $P_{1} P_{2}^{\prime} P_{3}^{\prime}=\left\langle 18^{\prime}, 14^{\prime}, 8, \overline{6}, 5,3^{2}, 1^{6}\right\rangle$ remains $F$-flat.

As in the previous example, there is also the possibility of a cancelation among different $F$-terms. In general, $P_{1} P_{2} P_{3}$ would be lifted by the same potential terms in (60), but some particular choice of VEVs leaves $V_{F}=0$. More precisely, solving the $D$-term constraints gives 


$$
\begin{aligned}
\left|\varphi_{1}\right|^{2} & =2 x^{2}, & & \left|\varphi_{8}\right|^{2}=x^{2}-\left|\psi_{1}\right|^{2} \\
\left|\varphi_{3}\right|^{2} & =\left|\psi_{1}\right|^{2}, & & \left|\varphi_{14}\right|^{2}=\left|\psi_{2}\right|^{2}, \\
\left|\varphi_{5}\right|^{2} & =\left|\psi_{1}\right|^{2}-\left|\psi_{2}\right|^{2}, & & \left|\varphi_{18}\right|^{2}=\left|\psi_{1}\right|^{2}-\left|\psi_{2}\right|^{2} . \\
\left|\varphi_{\overline{6}}\right|^{2} & =\left|\psi_{2}\right|^{2}, & &
\end{aligned}
$$

The choice $\left|\psi_{1}\right|^{2}=2\left|\psi_{2}\right|^{2}$ will give $V_{F}=0$ when the VEVs have the proper signs. In this case, we are left with a one-dimensional direction (parameterized by, say, $\left|\psi_{2}\right|^{2}$ ) $F$-flat to all orders. The particular point $\left|\psi_{2}\right|^{2}=x^{2} / 2$ gives $\left|\varphi_{8}\right|^{2}=0$ and corresponds to the flat direction $P_{2} P_{3}$ discussed before.

- $P_{4}\left(\Pi_{i} P_{\alpha}\right)$. The structure of the superpotential terms that lift $P_{4}$ is such that this type of $D$-flat directions is always lifted (a cancelation of different $F$-terms as in the examples above cannot be enforced). Moreover, some of the possible combinations, like $P_{4} P_{5}$, are type-A as they contain the invariant $\langle 6, \overline{6}\rangle$.

- $P_{5}\left(\Pi_{i} P_{\alpha}\right)$. Same as above.

A list of type-B flat directions which remain flat to all orders is presented in Table IV. The second column gives the dimensionality (a zero entry means that all field VEVs are determined in terms of the Fayet-Iliopoulos term) and the second gives the number of nonanomalous $U(1)$ 's broken along the corresponding direction. Those directions which are flat after imposing $F$-term constraints are indicated by $\left.\right|_{F}$. All type-B flat directions are trivially built out of the zero-dimensional ones in Table IV by addition of the primed copies of some fields. Some examples are already shown in that Table. Addition of a primed field to a given direction has the effect of increasing its dimensionality by one while the number of broken $U(1)$ 's is the same. For example, the addition of $S_{8}^{\prime}$ to $P_{1}$ results in the direction $P_{1} P_{1}^{\prime}$ and, as is shown in Table IV, the above rule is verified.

We can summarize our results by noting that all of the above flat directions are particular cases of the type-B flat direction

$$
\left.P_{1} P_{1}^{\prime} P_{2}^{\prime} P_{2}^{\prime \prime \prime} P_{3}^{\prime} P_{3}^{\prime \prime \prime}\right|_{F}
$$

This direction is five-dimensional and all other type-B flat directions can be viewed as particular sub-spaces of it, generated by setting some of the VEVs in (64) to zero or imposing some extra relation among them.

In other words, the moduli space of type-B flat directions is a subspace of the twelvedimensional field space

$$
\left\{S_{1}, S_{3}, S_{5}, S_{5}^{\prime}, \bar{S}_{6}, \bar{S}_{6}^{\prime}, S_{8}, S_{8}^{\prime}, S_{14}, S_{14}^{\prime}, S_{18}, S_{18}^{\prime}\right\}
$$

obtained by imposing the constraints from $D$-flatness ( 4 independent non-anomalous constraints plus the anomalous one) and $F$-flatness (the 2 conditions $\varphi_{14} \varphi_{\overline{6}}+\varphi_{18} \varphi_{5}=0$ and $\left.\varphi_{14} \varphi_{6}^{\prime}+\varphi_{18} \varphi_{5}^{\prime}=0\right)$. 


\section{SUMMARY AND CONCLUSIONS}

In this paper, we have developed techniques to classify systematically the $D$ - and $F$-flat directions for perturbative heterotic superstring vacua with $N=1$ supersymmetry and an anomalous $U(1)$ in the gauge group. At genus-one the superstring theory generates a FayetIliopoulos $D$-term $\xi \propto M_{\text {string }}^{2} /\left(192 \pi^{2}\right) \operatorname{Tr} Q_{A}$ [14,15]. The presence of this term triggers non-zero vacuum expectation values $(\mathrm{VEVs})$ for certain massless multiplets in such a way that the anomalous $U(1)$ is broken while maintaining the $D$-and $F$-flatness of the effective theory. Thus, the superstring vacuum is restabilized [14 16 and supersymmetry remains unbroken.

The analysis developed in this paper focuses on classification of the $D$ - and $F$-flat directions involving the non-Abelian singlets only; the results are illustrated with the explicit construction of flat directions for a particular string model, i.e., Model 5 of [7], which is based on the free fermionic construction.

The analysis was set up along the following stages:

\section{D-flatness}

- Non-anomalous $U(1)$ 's. First, the $D$-flat directions associated with the nonanomalous $U(1)$ 's are determined, by making use of the one-to-one correspondence of the $D$-flat directions with the holomorphic gauge-invariant monomials (HIM) of chiral superfields, constructed from the non-Abelian singlets ${ }^{⿴ 囗 十}$. We determined the moduli space of all such flat directions. Its dimension is $d \equiv \operatorname{dim} \mathcal{M}=N^{*}-\operatorname{Rank}\left[Q_{i}^{(a)}\right]^{*}$, where $N^{*}$ is the number of SM singlet fields that enter HIM's, and Rank $\left[Q_{i}^{(a)}\right]^{*}$ is the rank of the $N^{*} \times m^{*}$ charge matrix of the $N^{*}$ fields, which have non-zero charges under $m^{*}$ non-anomalous $U(1)^{(a)}$ factors. (The latter matrix is the one associated with $m^{*}$ $D_{(a)}$-flatness conditions for $N^{*}$ fields).

The construction of all flat directions from a basis of $d$ independent HIM's that characterizes the moduli space requires both multiplication and division of the elements of the basis, making it cumbersome to scan the whole moduli space. This difficulty is avoided if one considers another useful set, referred to as a superbasis, which is the set of all one-dimensional HIM's (see Table III for the chosen model). Every D-flat direction can then be obtained as a product of elements in this superbasis, so that positivity of the VEV squares is automatic and the contents of the moduli space are more clearly displayed. Such a set can be obtained by an automated computer search.

- Anomalous $U(1)$. The flatness of the $D$-term associated with the anomalous $U(1)$ requires that the Fayet-Iliopoulos term $\xi$ is balanced against the contribution to $D_{A}$ from the non-Abelian singlets $\varphi_{i}$, such that $D_{A}=\sum_{i} Q_{i}^{A}\left|\varphi_{i}\right|^{2}+\xi=0$. We classify the

\footnotetext{
${ }^{14}$ The fact that each chiral superfield enters the HIM's with a positive power is equivalent to ensuring that a particular $D$-flat direction involves VEVs of the fields whose absolute value-squared is positive.
} 
HIM's according to the sign of their contribution to the $D_{A}$-term, that is, according to their anomalous charge. They fall into three classes: their anomalous charge has a sign (i) equal to $-\operatorname{sign} \xi$, (ii) zero, (iii) equal to $+\operatorname{sign} \xi$. To ensure the $D_{A^{-}}$-flatness constraint, the (super)basis should necessarily contain one or more HIM's belonging to class (i). If this is not the case then the $D_{A}$-flatness constraint cannot be satisfied, i.e., there is a "no-go theorem" for the restabilization of the string vacuum via VEVs of the non-Abelian singlets $s^{\text {to }}$. In this case, the procedure necessarily requires non-zero VEVs for fields that transform under the non-Abelian gauge factors, further reducing the rank of the gauge group.

$D_{A}$-flat directions must therefore contain elements of the the superbasis that belong to class (i). However, they can be multiplied by the elements of the basis in class (ii) and/or class(iii), as long as the resulting HIM also belongs to class (i).

\section{F-flatness}

$F$-flatness requires $\partial W / \partial \Phi_{i}=0, W=0$ for all massless chiral superfields $\Phi_{i}$ of the model. The $D$-flat directions can be lifted due to the following two types of terms in the superpotential:

- $\mathbf{W}_{\mathbf{A}}$-Superpotential. Those are terms in the superpotential that include only the non-Abelian singlets which acquire non-zero VEVs along some direction (while ensuring the $D$-flatness of the effective theory). Products of elements of the superbasis which belong to class (ii) (their contribution to $D_{A}$ is zero) are not forbidden by gauge invariance, and thus they may appear in $W_{A}$. In certain cases one may be able to show that a stringy (world-sheet) symmetry ensures that a particular HIM element (with zero contribution to $D_{A}$ ) and all its positive powers are absent. However, in general conformal field theory techniques may not be powerful enough to determine the absence of all such terms. We therefore took a "conservative" approach that all such HIM elements could appear in $W_{A}$ at some order and could thus lift the (type-A) $D$-flat direction (barring cancelation among different terms of that type). Consequently, we remove the elements in the superbasis that belong to class (ii). (It is of course possible that there are additional flat directions that are missed by our conservative approach). One can also show (see Section V) that elements of the superbasis belonging to class (iii) should also be removed, because they can generate terms in class (ii) when they are multiplied with appropriate powers of the superbasis elements in class (i). Therefore, the remaining elements in the superbasis, which ensure type-A $F$-flatness to all orders, consist of the subset belonging to class (i) only.

- $\mathbf{W}_{\mathrm{B}}$-Superpotential. Those are the terms in the superpotential which contain one power of a non-Abelian singlet field $\Psi_{i}$ with zero VEV, while all the other fields have

\footnotetext{
${ }^{15}$ Whether or not this is the case can be discerned already at the level of the elementary fields, without the need of computing the superbasis, as is discussed in section IV.
} 
non-zero VEVs along the given direction. Such terms, if present, can lift (type-B) $F$-flat directions (i.e. those directions for which no $W_{A}$ terms are possible). Gauge invariance constrains the number of allowed terms in $W_{B}$ to a finite number. By doing a string calculation, one can then check explicitly whether these terms are absent thus ensuring the type-B $F$-flatness conditions $\square$.

Combining class (i) elements of the superbasis and checking the superpotential, one can generate all type-B directions which are $D$ - and $F$-flat to all orders.

In conclusion, we developed techniques that set the stage to classify systematically the $D$ and $F$-flat directions involving nonabelian singlet fields only for a large class of perturbative string vacua with an anomalous $U(1)$. In a subsequent paper [17], these techniques are applied to a class of models based on the free fermionic construction, which possess three ordinary families and the standard model gauge group in the observable sector.

\section{ACKNOWLEDGMENTS}

This work was supported in part by U.S. Department of Energy Grant No. DOE-EY76-02-3071.

\footnotetext{
${ }^{16}$ In certain cases the type-B $F$-flat directions can also be obtained by employing cancelation of different terms in $W_{B}$ (See Section V).
} 


\section{REFERENCES}

[1] L. Dixon, J. A. Harvey, C. Vafa and E. Witten, Nucl. Phys. B261 (1985) 678, Nucl. Phys. B274 (1986) 285.

[2] L. Ibáñez, H.P. Nilles and F. Quevedo, Phys. Lett. B187 (1987) 25; Phys. Lett. B192 (1987) 332.

[3] L. Ibáñez, J.E. Kim, H.P. Nilles and F. Quevedo, Phys. Lett. B191 (1987) 282; J.A. Casas and C. Muñoz, Phys. Lett. B209 (1988) 214; Phys. Lett. B214 (1988) 157; J.A. Casas, E. Katehou and C. Muñoz, Nucl. Phys. B317 (1989) 171; A. Font, L. Ibáñez, H.P. Nilles and F. Quevedo, Phys. Lett. B210 (1988) 101; A. Chamseddine and M. Quirós, Phys. Lett. B212 (1988) 343; Nucl. Phys. B316 (1989) 101; A. Font, L. Ibáñez, F. Quevedo and A. Sierra, Nucl. Phys. B331 (1990) 421.

[4] B. Greene, K. Kirlin, P. Miron and G.G. Ross, Nucl. Phys. B278 (1986) 667; Nucl. Phys. B292 (1987) 606

[5] I. Antoniadis, C. Bachas and C. Kounnas, Nucl. Phys. B289 (1987) 87; H. Kawai, D.C. Lewellen and S.H.-H. Tye, Phys. Rev. Lett. 57 (1986) 1832, Nucl. Phys. B288 (1987) 1, Phys. Rev. D34 (1986) 3794; H. Kawai, D. Lewellen, J.A. Schwartz and S.-H. Tye, Nucl. Phys. B299 (1988) 431; I. Antoniadis and C. Bachas, Nucl. Phys. B298 (1988) 586.

[6] I. Antoniadis, J. Ellis, J. Hagelin and D. Nanopoulos, Phys. Lett. B231 (1989) 65; J. López, D. Nanopoulos and K. Yuan, Nucl. Phys. B399 (1993) 654; A. Faraggi, Phys. Lett. B274 (1992) 47; Nucl. Phys. B387 (1992) 239; S. Chaudhuri, S.-W. Chung, G. Hockney and J. Lykken, Nucl. Phys. B456 (1995) 89; J. López, D. Nanopoulos and A. Zichichi, Phys. Rev. D53 (1996) 5253.

[7] S. Chaudhuri, G. Hockney and J. Lykken, Nucl. Phys. B469 (1996) 357.

[8] B.E. Nilsson, P. Roberts and P. Salomonson, Phys. Lett. B222 (1989) 35.

[9] A. Font, L. Ibáñez, M. Mondragón, G.G. Ross and F. Quevedo, Phys. Lett. B227 (1989) 34; P. Berglund, C. Johnson, S. Kachru and P. Zaugg, Nucl. Phys. B460 (1996) 252.

[10] L. Dixon, E. Martinec, D. Friedan and S. Shenker, Nucl. Phys. B282 (1987) 13.

[11] L. Dixon and M. Cvetič, unpublished; M. Cvetič, in Proceedings of Superstrings, Cosmology, and Composite Structures, College Park, Maryland, March 1987, S. J. Gates and R. Mohapatra, eds.(World Scientific 1987); M. Cvetič, Phys. Rev. Lett. 59 (1987) 2829.

[12] M. Cvetič and P. Langacker, Phys. Rev. D54 (1996) 3570; Mod. Phys. Lett. A11 (1996) 1247 and hep-ph/9707451].

[13] M. Cvetič, D.A. Demir, J.R. Espinosa, L. Everett and P. Langacker, Phys. Rev. D56 (1997) 2861; G. Cleaver, M. Cvetič, J.R. Espinosa, L. Everett and P. Langacker, hepph/9705391].

[14] M. Dine, N. Seiberg and E. Witten, Nucl. Phys. B289 (1986) 585.

[15] J. Atick, L. Dixon and A. Sen, Nucl. Phys. B292 (1987) 109; M. Dine, I. Ichinose, and N. Seiberg, Nucl. Phys. B293 (1987) 253; M. Dine and C. Lee, Nucl. Phys. B336 (1990) 317.

[16] L. Dixon and V. Kaplunovsky, unpublished.

[17] G. Cleaver et al., in preparation.

[18] G. Cleaver and A.E. Faraggi, UFIFT-HEP-97-28, UPR-0773-T, hep-ph/9711339. 
[19] T. Kobayashi and H. Nakano, Nucl. Phys. B496 (1997) 103.

[20] L. Ibáñez and G.G. Ross, Phys. Lett. B332 (1994) 100; P. Binétruy and P. Ramond, Phys. Lett. B350 (1995) 49; P. Binétruy, S. Lavignac and P. Ramond, Nucl. Phys. B477 (1996) 353; E. Dudas, C. Grojean, S. Pokorski and C. Savoy, Nucl. Phys. B481 (1996) 85; E. Dudas, S. Pokorski and C. Savoy, Phys. Lett. B356 (1995) 45; T. Kobayashi, Phys. Lett. B354 (1995) 264, Phys. Lett. B358 (1995) 253; T. Kobayashi and Z.Z. Xing, Mod. Phys. Lett. A12 (1997) 561.

[21] Y. Nir, Phys. Lett. B354 (1995) 107; V. Jain and R. Shrock, Phys. Lett. B352 (1995) 83.

[22] J.A. Casas and C. Muñoz, Phys. Lett. B216 (1989) 37; J.A. Casas, J.M. Moreno, C. Muñoz and M. Quirós, Nucl. Phys. B328 (1989) 272; P. Binétruy and G. Dvali, Phys. Lett. B388 (1996) 241; E. Halyo, Phys. Lett. B387 (1996) 43.

[23] P. Binétruy and E. Dudas, Phys. Lett. B389 (1996) 503.

[24] G. Dvali and A. Pomarol, Phys. Rev. Lett. 77 (1996) 3728; R.N. Mohapatra and A. Riotto, Phys. Rev. D55 (1997) 4262.

[25] L. Ibáñez, Phys. Lett. B303 (1993) 55.

[26] F. Buccella, J.-P. Derendinger, C. Savoy and S. Ferrara, Phys. Lett. B115 (1982) 375.

[27] See e.g. M.A. Luty and W. Taylor IV, Phys. Rev. D53, (1996) 3399 and references therein.

[28] T. Gherghetta, C. Kolda and S. Martin, Nucl. Phys. B468 (1996) 37.

[29] P. Binétruy, N. Irges, S. Lavignac and P. Ramond, Phys. Lett. B403 (1997) 38.

[30] See e.g. J.M. Thomas, Theory of Equations, MacGraw-Hill. Theorem 27.7, p.49. 


\begin{tabular}{|c|c|ccccccc|c|c|}
\hline \hline $\begin{array}{c}\text { NA } \\
\text { Singlet }\end{array}$ & $\begin{array}{c}\text { Vector- } \\
\text { like } \\
+/-\end{array}$ & $Q_{A}$ & $Q_{1}$ & $Q_{2}$ & $Q_{3}$ & $Q_{4}$ & $Q_{5}$ & $Q_{6}$ & $Q_{Y}$ & $\hat{Q}$ \\
\hline \hline$S_{1}$ & $\sqrt{ }$ & 28 & 0 & 0 & 0 & 0 & 12 & 12 & 0 & 32 \\
$S_{2}$ & $\sqrt{ }$ & 8 & 0 & 4 & 16 & 0 & -8 & -24 & -1 & 0 \\
$S_{3}$ & $\sqrt{ }$ & 8 & 0 & 0 & -16 & 4 & -8 & -24 & 0 & 0 \\
$S_{4}$ & $\sqrt{ }$ & 0 & 4 & 0 & 0 & 0 & 0 & 0 & 0 & 0 \\
$S_{5}{ }^{(\prime)}$ & $V^{(\prime)}$ & 0 & 2 & 4 & 0 & -2 & -16 & 0 & 0 & 0 \\
$S_{6}{ }^{(\prime)}$ & $\mathcal{V}^{(\prime)}$ & 0 & 2 & -4 & 0 & 2 & 16 & 0 & 0 & 0 \\
$S_{7}$ & & 20 & 0 & -2 & 8 & 2 & 12 & -60 & -1 & 0 \\
$S_{8}{ }^{(\prime)}$ & & 8 & 0 & 0 & 0 & 0 & -24 & -24 & 0 & 0 \\
$S_{9}$ & & 4 & -4 & -2 & -24 & -2 & -4 & -12 & 1 & 0 \\
$S_{10}$ & & 4 & 4 & -2 & -24 & -2 & -4 & -12 & 1 & 0 \\
$S_{11}{ }^{(\prime)}$ & & 4 & 0 & 2 & 8 & -6 & -4 & -12 & 0 & 0 \\
$S_{12}$ & & 4 & 0 & 2 & -24 & 2 & 28 & -12 & 0 & 0 \\
$S_{13}$ & & 0 & 2 & 0 & -16 & -2 & -32 & 0 & 1 & 0 \\
$S_{14}{ }^{(\prime)}$ & & 0 & 2 & -4 & 16 & -2 & 0 & 0 & 0 & 0 \\
$S_{15}{ }^{(\prime)}$ & & 0 & 0 & 0 & -16 & 4 & 16 & 0 & 0 & 0 \\
$S_{16}{ }^{(\prime)}$ & & 0 & 0 & 4 & 16 & 0 & 16 & 0 & -1 & 0 \\
$S_{17}$ & & 0 & -2 & 0 & -16 & -2 & -32 & 0 & 1 & 0 \\
$S_{18}{ }^{(\prime)}$ & & 0 & -2 & -4 & 16 & -2 & 0 & 0 & 0 & 0 \\
$S_{19}{ }^{(\prime)}$ & & -24 & 0 & 2 & -8 & -2 & 0 & -24 & 0 & -32 \\
$S_{20}$ & & -12 & 0 & -2 & 8 & 2 & 12 & 36 & 0 & 0 \\
$S_{21}{ }^{(\prime)}$ & & -8 & 0 & -4 & 0 & -4 & -8 & 24 & 1 & 0 \\
$S_{22}$ & & -8 & 2 & 4 & 0 & -2 & 8 & 24 & 0 & 0 \\
$S_{23}$ & & -8 & -2 & 4 & 0 & -2 & 8 & 24 & 0 & 0 \\
$S_{24}{ }^{(\prime)}$ & & -4 & 0 & 2 & 24 & 2 & 4 & 12 & -1 & 0 \\
$S_{25}{ }^{(\prime)}$ & & -4 & 0 & -2 & -8 & -6 & 4 & 12 & 1 & 0 \\
\hline \hline
\end{tabular}

Table I: List of non-Abelian singlet fields in the model with their charges under the $U(1)$ gauge groups, hypercharge as defined in eq.(11) and $\hat{Q}=Q_{A}+Q_{6} / 3$. A prime in parentheses indicates that there is an extra copy of the field with exactly the same $U(1)$ charges. A $\sqrt{ }$ in the second column indicates that there is an extra copy of the field with exactly opposite $U(1)$ charges. 


\begin{tabular}{|l|c||l|c|}
\hline \hline BASIS A & $\hat{Q}$ & BASIS B & $\hat{Q}$ \\
\hline \hline$M_{1}=\langle 1, \overline{1}\rangle$ & 0 & $P_{1}=\left\langle 8,1^{2}\right\rangle$ & 64 \\
$M_{2}=\langle 3, \overline{3}\rangle$ & 0 & $P_{2}=\left\langle 14, \overline{6}, 3,1^{2}\right\rangle$ & 64 \\
$M_{3}=\langle 4, \overline{4}\rangle$ & 0 & $P_{3}=\left\langle 18,5,3,1^{2}\right\rangle$ & 64 \\
$M_{4}=\langle 5, \overline{5}\rangle$ & 0 & $P_{4}=\left\langle 14,5, \overline{4}, 3,1^{2}\right\rangle$ & 64 \\
$M_{5}=\langle 6, \overline{6}\rangle$ & 0 & $P_{5}=\left\langle 18, \overline{6}, 4,3,1^{2}\right\rangle$ & 64 \\
$M_{6}=\left\langle 8,1^{2}\right\rangle$ & 64 & $N_{1}=\langle 1, \overline{1}\rangle$ & 0 \\
$M_{7}=\langle 20,11,3\rangle$ & 0 & $N_{2}=\langle 3, \overline{3}\rangle$ & 0 \\
$M_{8}=\langle 23,14,3\rangle$ & 0 & $N_{3}=\langle 5, \overline{5}\rangle$ & 0 \\
$M_{9}=\langle 22,18,3\rangle$ & 0 & $N_{4}=\langle 6, \overline{6}\rangle$ & 0 \\
$M_{10}=\langle\overline{6}, \overline{5}, 4\rangle$ & 0 & $N_{5}=\langle 20,11,3\rangle$ & 0 \\
$M_{11}=\langle 18,15,5\rangle$ & 0 & $N_{6}=\langle 23,14,3\rangle$ & 0 \\
$M_{12}=\langle 23,8,6\rangle$ & 0 & $N_{7}=\langle 22,18,3\rangle$ & 0 \\
$M_{13}=\langle 15, \overline{3}, 8\rangle$ & 0 & $N_{8}=\langle 18,15,5\rangle$ & 0 \\
$M_{14}=\langle 20,19, \overline{1}\rangle$ & -64 & $N_{9}=\langle 20,19, \overline{1}\rangle$ & -64 \\
$M_{15}=\langle 20,18,12,8,5\rangle$ & 0 & $N_{10}=\langle 20,18,12,8,5\rangle$ & 0 \\
\hline \hline
\end{tabular}

Table II: Two different bases of the moduli space of non-anomalous $D$-flat directions of the model. Each element corresponds to a holomorphic monomial which is gauge invariant under the non-anomalous $U(1)$ 's. The anomalous charge is given by $\hat{Q}$. 


\begin{tabular}{|c|c|c|c|c|c|}
\hline SUPERBASIS & $\hat{Q}$ & $R_{25}=\left\langle 23, \overline{5}, 4, \overline{1}^{2}\right\rangle$ & -64 & $R_{50}=\langle 22,14, \overline{6}, \overline{5}, 3\rangle$ & 0 \\
\hline$R_{1}=\langle 1, \overline{1}\rangle$ & 0 & $R_{26}=\left\langle 22,6, \overline{4}, \overline{1}^{2}\right\rangle$ & -64 & $R_{51}=\langle 23,18,6,5,3\rangle$ & 0 \\
\hline$R_{2}=\langle 3, \overline{3}\rangle$ & 0 & $R_{27}=\left\langle 20^{2}, 12,11, \overline{1}^{4}\right\rangle$ & -128 & $R_{52}=\left\langle 20,18^{2}, 12,5^{2}, 3\right\rangle$ & 0 \\
\hline$R_{3}=\langle 4, \overline{4}\rangle$ & 0 & $R_{28}=\left\langle 20,15,11, \overline{1}^{2}\right\rangle$ & -64 & $R_{53}=\left\langle 20^{2}, 19^{2}, 18,5,3\right\rangle$ & -64 \\
\hline$R_{4}=\langle\overline{5}, \overline{5}\rangle$ & 0 & $R_{29}=\left\langle 23,15,14, \overline{1}^{2}\right\rangle$ & -64 & $R_{54}=\left\langle 20,14^{2}, 12, \overline{6}^{2}, 3\right\rangle$ & 0 \\
\hline$R_{5}=\langle 6, \overline{6}\rangle$ & 0 & $R_{30}=\left\langle 22,18,15, \overline{1}^{2}\right\rangle$ & -64 & $R_{55}=\left\langle 20^{2}, 19^{2}, 14, \overline{6}, 3\right\rangle$ & -64 \\
\hline$R_{6}=\left\langle 8,1^{2}\right\rangle$ & 64 & $R_{31}=\langle 22,14, \overline{4}, 3\rangle$ & 0 & $R_{56}=\langle 22,15,14,8, \overline{4}\rangle$ & 0 \\
\hline$R_{7}=\left\langle 15, \overline{3}, \overline{1}^{2}\right\rangle$ & -64 & $R_{32}=\langle 23,18,4,3\rangle$ & 0 & $R_{57}=\langle 23,18,15,8,4\rangle$ & 0 \\
\hline$R_{8}=\left\langle 22, \overline{5}, \overline{1}^{2}\right\rangle$ & -64 & $R_{33}=\langle 15,14,5, \overline{4}\rangle$ & 0 & $R_{58}=\langle 20,18,12,8,5\rangle$ & 0 \\
\hline$R_{9}=\left\langle 23, \overline{6}, \overline{1}^{2}\right\rangle$ & -64 & $R_{34}=\langle 18,15, \overline{6}, 4\rangle$ & 0 & $R_{59}=\langle 20,14,12,8, \overline{6}\rangle$ & 0 \\
\hline$R_{10}=\langle 20,19, \overline{1}\rangle$ & -64 & $R_{35}=\left\langle 20,12,8^{2}, \overline{3}\right\rangle$ & 0 & $R_{60}=\left\langle 23,20,14,12,8^{2}\right\rangle$ & 0 \\
\hline$R_{11}=\langle 20,11,3\rangle$ & 0 & $R_{36}=\langle 23,8, \overline{5}, 4\rangle$ & 0 & $R_{61}=\left\langle 22,20,18,12,8^{2}\right\rangle$ & 0 \\
\hline$R_{12}=\langle 23,14,3\rangle$ & 0 & $R_{37}=\langle 22,8,6, \overline{4}\rangle$ & 0 & $R_{62}=\left\langle 20,14,12,5, \overline{4}, \overline{1}^{2}\right\rangle$ & -64 \\
\hline$R_{13}=\langle 22,18,3\rangle$ & 0 & $R_{38}=\left\langle 20^{2}, 12,11,8^{2}\right\rangle$ & 0 & $R_{63}=\left\langle 20,18,12, \overline{6}, 4, \overline{1}^{2}\right\rangle$ & -64 \\
\hline$R_{14}=\langle\overline{6}, \overline{5}, 4\rangle$ & 0 & $R_{39}=\langle 20,15,11,8\rangle$ & 0 & $R_{64}=\left\langle 22,20,14,12, \overline{4}, \overline{1}^{4}\right\rangle$ & -128 \\
\hline$R_{15}=\langle 6,5, \overline{4}\rangle$ & 0 & $R_{40}=\langle 23,15,14,8\rangle$ & 0 & $R_{65}=\left\langle 23,20,18,12,4, \overline{1}^{4}\right\rangle$ & -128 \\
\hline$R_{16}=\langle 18,15,5\rangle$ & 0 & $R_{41}=\langle 22,18,15,8\rangle$ & 0 & $R_{66}=\left\langle 20,14^{2}, 12,5^{2}, \overline{4}^{2}, 3\right\rangle$ & 0 \\
\hline$R_{17}=\langle 15,14, \overline{6}\rangle$ & 0 & $R_{42}=\left\langle 14,5, \overline{4}, 3,1^{2}\right\rangle$ & 64 & $R_{67}=\left\langle 20^{2}, 19^{2}, 14,5, \overline{4}, 3\right\rangle$ & -64 \\
\hline$R_{18}=\langle 15,8, \overline{3}\rangle$ & 0 & $R_{43}=\left\langle 18, \overline{6}, 4,3,1^{2}\right\rangle$ & 64 & $R_{68}=\left\langle 20,18^{2}, 12, \overline{6}^{2}, 4^{2}, 3\right\rangle$ & 0 \\
\hline$R_{19}=\langle 22,8, \overline{5}\rangle$ & 0 & $R_{44}=\left\langle 22,15,14, \overline{4}, \overline{1}^{2}\right\rangle$ & -64 & $R_{69}=\left\langle 20^{2}, 19^{2}, 18, \overline{6}, 4,3\right\rangle$ & -64 \\
\hline$R_{20}=\left\langle 20^{2}, 19^{2}, 8\right\rangle$ & -64 & $R_{45}=\left\langle 23,18,15,4, \overline{1}^{2}\right\rangle$ & -64 & $R_{70}=\langle 20,14,12,8,5, \overline{4}\rangle$ & 0 \\
\hline$R_{21}=\langle 23,8,6\rangle$ & 0 & $R_{46}=\left\langle 20,18,12,5, \overline{1}^{2}\right\rangle$ & -64 & $R_{71}=\langle 20,18,12,8, \overline{6}, 4\rangle$ & 0 \\
\hline$R_{23}=\left\langle 14, \overline{6}, 3,1^{2}\right\rangle$ & 64 & $R_{47}=\left\langle 20,14,12, \overline{6}, \overline{1}^{2}\right\rangle$ & -64 & $R_{72}=\left\langle 22,20,14,12,8^{2}, \overline{4}\right\rangle$ & 0 \\
\hline$R_{22}=\left\langle 18,5,3,1^{2}\right\rangle$ & 64 & $R_{48}=\left\langle 23,20,14,12, \overline{1}^{4}\right\rangle$ & -128 & $R_{73}=\left\langle 23,20,18,12,8^{2}, 4\right\rangle$ & 0 \\
\hline$R_{24}=\left\langle 20,12, \overline{3}, \overline{1}^{4}\right\rangle$ & -128 & $R_{49}=\left\langle 22,20,18,12, \overline{1}^{4}\right\rangle$ & -128 & -------- & - \\
\hline
\end{tabular}

Table III: Complete list of one-dimensional non-anomalous $D$-flat directions of the model with their corresponding anomalous charges.

\begin{tabular}{|l|c|c|}
\hline \hline FLAT DIRECTION & Dim. & $\# U(1)^{\prime} \mathrm{s}$ \\
\hline \hline$P_{1}^{(\prime)}=\left\langle 8^{(\prime)}, 1^{2}\right\rangle$ & 0 & 1 \\
$P_{2}^{\prime(\prime)}=\left\langle 14^{\prime}, \overline{6}^{(\prime)}, 3,1^{2}\right\rangle$ & 0 & 3 \\
$P_{3}^{\prime(\prime)}=\left\langle 18^{\prime}, 5^{(\prime)}, 3,1^{2}\right\rangle$ & 0 & 3 \\
$\left.P_{2} P_{3}\right|_{F},\left.P_{2}^{\prime \prime \prime} P_{3}^{\prime \prime \prime} \equiv\left\langle 18,14, \overline{6}^{\prime}, 5^{\prime}, 3^{2}, 1^{4}\right\rangle\right|_{F}$ & 0 & 4 \\
$P_{1} P_{1}^{\prime}$ & 1 & 1 \\
$P_{2}^{\prime} P_{2}^{\prime \prime}, P_{3}^{\prime} P_{3}^{\prime \prime}$ & 1 & 3 \\
$P_{1}^{(\prime)} P_{2}^{\prime(\prime)}, P_{1}^{(\prime)} P_{3}^{\prime(\prime)}$ & 1 & 3 \\
$P_{2}^{\prime(\prime)} P_{3}^{\prime(\prime)}$ & 1 & 4 \\
$\left.P_{1}^{(\prime)} P_{2} P_{3}\right|_{F},\left.P_{1}^{(\prime)} P_{2}^{\prime \prime \prime} P_{3}^{\prime \prime \prime}\right|_{F}$ & 1 & 4 \\
\hline \hline
\end{tabular}

Table IV: List of type-B $D$-flat directions which are $F$-flat to all orders for the model discussed. The dimension of the direction, after cancelation of the Fayet-Iliopoulos term, is indicated in the second column. The third column gives the number of non-anomalous $U(1)$ 's broken along the flat direction. 